\title{
Synthesis of some 1- and 2-carboxyalkyl substituted benzimidazoles and their derivatives
}

\author{
Kristina Mickevičienė • Aušra Voskienè • \\ Vytautas Mickevičius
}

Received: 5 December 2012/Accepted: 21 January 2013/Published online: 5 February 2013

(C) The Author(s) 2013. This article is published with open access at Springerlink.com

\begin{abstract}
Mono- and disubstituted benzimidazoles were synthesized during alkaline hydrolysis or reactions with ethyl chloroacetate of 1-phenyl substituted 4-(1H-benzimidazol-2-yl)-2-pyrrolidinones. The properties of the synthesized ethyl[2-(1-(substituted phenyl)-5-oxopyrrolidinyl-3-yl)-1H-benzimidazolyl]ethanoates have been investigated and their benzimidazolium chlorides, 1-carboxymethyl benzimidazoles, condensation products of 2-\{2-[1-(3-methylphenyl)-5-oxo-3-pyrrolidinyl]-1H-benzimidazol-1-yl $\}$ acetohydrazide with various aromatic aldehydes and aliphatic ketones have been obtained.
\end{abstract}

Keywords Heterocycles · Benzimidazoles - Pyrrolidinone - Carbohydrazide · Condensation

\section{Introduction}

Benzimidazole heterosystems are present in many natural and synthetic biological activity structures and are of great interest in medical chemistry and pharmacology. Benzimidazole derivatives are distinguished for antimicrobial [1-4], antifungal [5-7], antiviral [8], anthelmintic [9, 10], antihypertensive [11], antihistaminic [12], analgesic [13], and anti-HIV [14] actions. Also, some of benzimidazoles are used in coordination chemistry [15, 16], in optoelectronics [17], etc. The aim of this study was to synthesize new potentially bioactive benzimidazole derivatives or its intermediates containing carboxyalkyl, hydrazone, pyrrole, and dimethylpyrazole fragments.

K. Mickevičienè · A. Voskienè · V. Mickevičius $(\bowtie)$

Department of Organic Chemistry, Kaunas University of Technology, Radvilenu pl. 19,

50254 Kaunas, Lithuania

e-mail: vymic@ktu.lt 


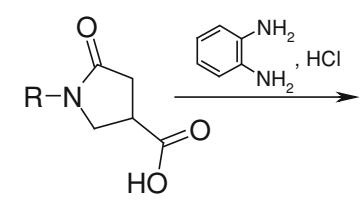

1a-1c

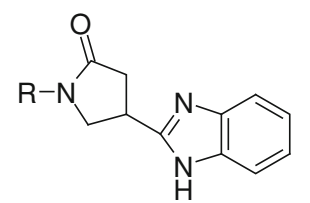

2a-2c

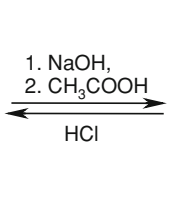<smiles>[R]NCC(C)c1nc2ccccc2[nH]1</smiles>

3a-3c

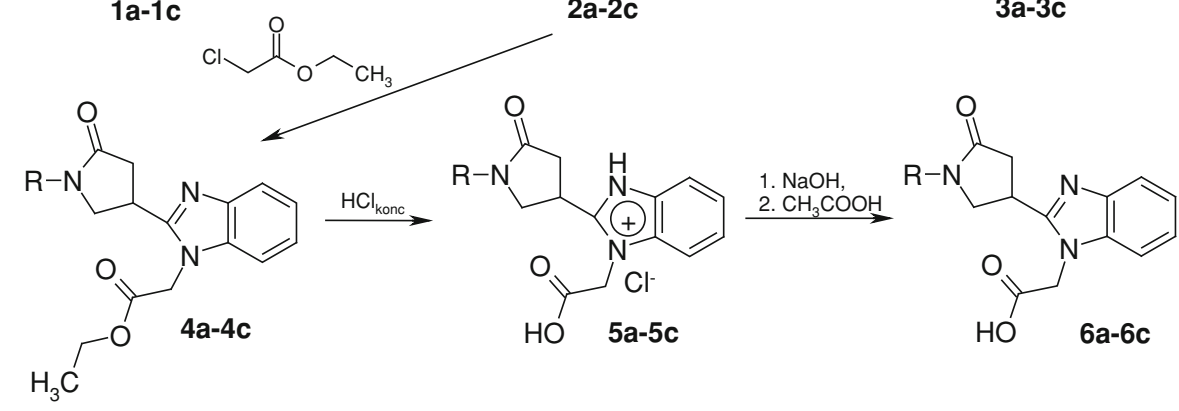

$$
\left.\mathbf{R}=\text { a) } 3-\mathrm{CH}_{3}-\mathrm{C}_{6} \mathrm{H}_{4} \text {, b) 2,5- }\left(\mathrm{CH}_{3}\right)_{2}-\mathrm{C}_{6} \mathrm{H}_{3}, \mathbf{c}\right) 2-\mathrm{CH}_{3}-5-\mathrm{Cl}_{-} \mathrm{C}_{6} \mathrm{H}_{3}
$$

Scheme 1 Synthesis of benzimidazole derivatives 2-6

\section{Results and discussion}

We report here on the synthesis of some new 2- and 1,2-substituted benzimidazoles prepared from 5-oxo-1-phenyl-3-pyrrolidinecarboxylic acids. One of the methods for the synthesis of a benzimidazole heterosystem is condensation of carboxylic acids with 1,2-diaminobenzenes. The target compounds were synthesized by the Phillips method (heating of both reagents in $4 \mathrm{M}$ hydrochloric acid); we obtained a sufficient yield of benzimidazoles (Scheme 1).

It is known that the 5-oxopyrrolidine cycle is not resistant to alkaline hydrolysis $[18,19]$. In the present work, sodium salts of 4-arylamino-3-(1H-benzimidazol-2yl)butanoic acids were formed by decomposition of the pyrrolidinone cycle of 1-aryl-3-(1H-benzimidazol-2-yl)-5-oxopyrrolidines 2a-2c in refluxing a $20 \%$ solution of sodium hydroxide. Acidification of the aqueous solutions of these salts with acetic acid up to $\mathrm{pH} 6$ gave stable 3-(1H-benzimidazol-2-yl)-4-arylaminobutanoic acids 3a-3c (Scheme 1). They were purified by a double precipitation from alkaline solution with acetic acid.

The opposite reaction of cyclization of the open-chain $\mathbf{3 a}-\mathbf{3} \mathbf{c}$ compounds to $\mathbf{2 a - 2} \mathbf{c}$ was also carried out by boiling $\gamma$-amino acids $\mathbf{3 a}-\mathbf{3 c}$ in diluted hydrochloric acid and subsequently neutralizing the reaction mixture with aqueous ammonia. The cyclic compounds $\mathbf{2 a - 2 \mathbf { c }}$ were obtained in a $91-95 \%$ yield.

The structural changes of series 3 compounds have been revealed by comparison of their ${ }^{13} \mathrm{C}$ NMR spectra with those of the corresponding compounds $\mathbf{2}$ containing a pyrrolidinone ring. The resonance at $\sim 175 \mathrm{ppm}$ clearly shows the presence of an open-chain compound. Chemical shifts of atoms C-2 and C-3 of these compounds are quite close - the difference is only 1.4-1.7 ppm, while in cyclic compounds it reaches up to 5-6.5 ppm. In ${ }^{1} \mathrm{H}$ NMR spectra, 3-(1H-benzimidazol-2-yl)-4-(substituted 
phenylamino)butanoic acids a broad 3a-3c singlet of $\mathrm{NH}$ in the region of 5.57-5.98 ppm confirmed the existence of open chain compounds. The broad absorption band characteristic of the $\mathrm{NH}$ and $\mathrm{OH}$ groups is observed in the region $2,840-3,430 \mathrm{~cm}^{-1}$ in the IR spectra of these compounds. It partially overlaps with the absorbtion bands of the aromatic system.

We investigated the alkylation reaction of benzimidazoles $\mathbf{3}$ with ethyl chloroacetate (Scheme 1). Substituted benzimidazole derivatives $\mathbf{4 a - 4 c}$ were synthesized by alkylation of 1-aryl-3-(1H-benzimidazol-2-yl)-5-oxopyrrolidines 3a-3c with ethyl chloroacetate in toluene in the presence of potassium carbonate, potassium hydroxide, and a catalytic amount of tetrabutylammonium iodide. Hydrolysis of the synthesized esters $\mathbf{4 a - 4} \mathbf{c}$ was carried out in refluxing concentrated hydrochloric acid. In these conditions, not only hydrolysis of the ester group took place but the corresponding benzimidazolium chlorides $5 \mathbf{a}-\mathbf{c}$ were also formed. They were converted to the respective bases $\mathbf{6 a - 6 c}$ by heating quaternary salts in a sodium hydroxide solution and then acidifying with acetic acid. Compounds $\mathbf{6 a - 6 c}$ were purified by dissolving them in a sodium alkaline solution, filtrating the solution, and acidifying the filtrate with acetic acid up to $\mathrm{pH}$. The IR, ${ }^{1} \mathrm{H}$ and ${ }^{13} \mathrm{C}$ NMR and mass spectra were in agreement with the suggested structures of compounds 4-6.

New hydrazones and azoles containing benzimidazole and pyrrolidinone moieties were synthesized from 2-\{2-[1-(3-methylphenyl)-5-oxo-3-pyrrolidinyl]-1H-benzimidazol-1-yl \}acetohydrazide (7) (Scheme 2). Carbohydrazide 7 was obtained by reaction of the ethyl ester $\mathbf{4 a}$ with hydrazine hydrate in refluxing 2-propanol. The hydrazones 8-12 were synthesized by condensation of carbohydrazide $\mathbf{7}$ with aromatic aldehydes or ketones - acetone and ethylmethylketone. Analysis of ${ }^{1} \mathrm{H}$ NMR spectra of 1-aryl-3-arylidenehydrazinocarbonyl-5-oxopyrrolidines 8-12 showed that a mixture of $E / Z$ rotamers exists in DMSO- $d_{6}$ solutions in which $Z$ isomer predominates due to a hindered rotation around the $\mathrm{CO}-\mathrm{NH}$ bond $[20,21]$.

During reactions of carbohydrazide 7 with 2,4-pentanedione or 2,5-hexanedione, performed with refluxing 2-propanol in the presence of a catalytic amount of acetic or hydrochloric acid, the N-substituted pyrrazole or pyrrole derivatives 13, 14 were synthesized. The structure of these compounds authenticates the spectral data. For example, the formation of a 2,5-methylpyrrole ring included in the $\mathbf{1 4}$ composition is displayed by the double-intensity resonances of $\mathrm{CH}$ at $109.7 \mathrm{ppm},=\mathrm{C}$ at $128.5 \mathrm{ppm}$, and $\mathrm{CH}_{3}$ at $11.0 \mathrm{ppm}$ in ${ }^{13} \mathrm{C}$ NMR spectra, and singlets at $2.01 \mathrm{ppm}$ $\left(\mathrm{CH}_{3}\right), 5.65 \mathrm{ppm}(=\mathrm{CH})$, and $11.20 \mathrm{ppm}(\mathrm{NH})$ in ${ }^{1} \mathrm{H}$ NMR spectra.

\section{Conclusion}

1-Phenyl substituted 4-(1H-benzimidazol-2-yl)-2-pyrrolidinones have been synthesized, their properties have been investigated, and it has been determined that during alkaline hydrolysis, the pyrrolidinone cycle cleaves forming sodium 3-(1H-benzimidazol-2-yl)-4-arylaminobutanoates which transform into 3-(1H-benzimidazol2-yl)-4-arylaminobutanoic acids when treated with acetic acid. By alkylation of the benzimidazole cycle with ethyl chloroacetate, $\mathrm{N}$-alkylated products are formed. The 


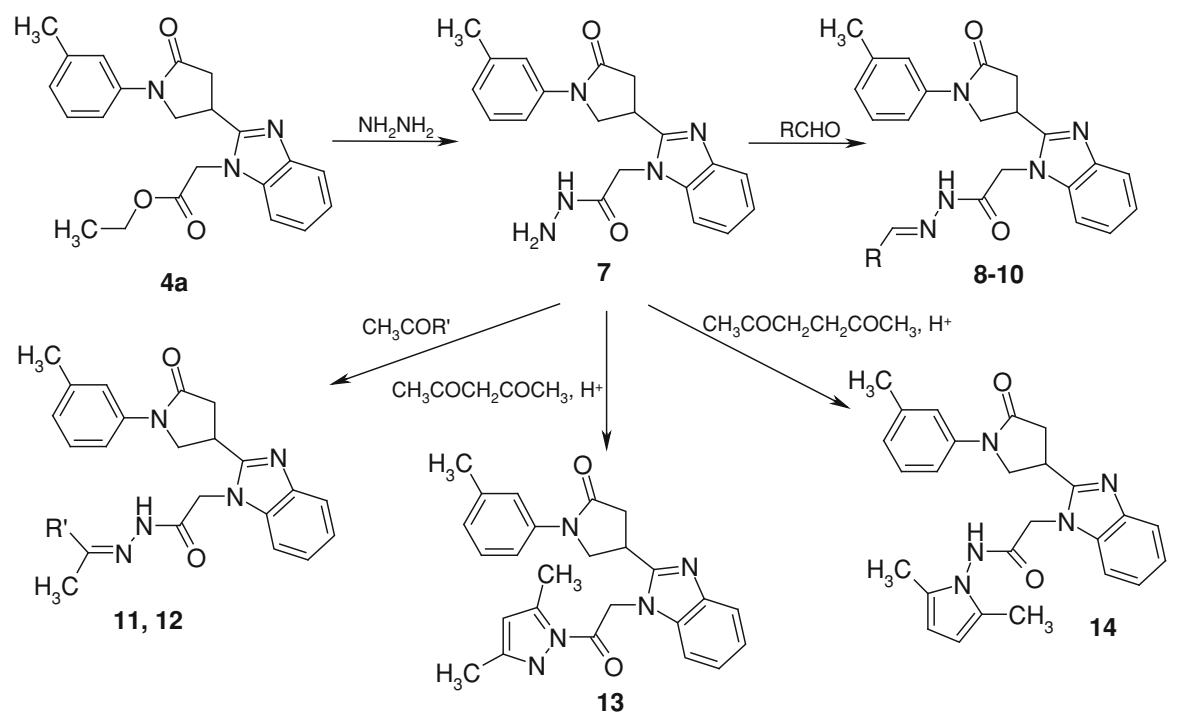

$\mathbf{R}=$ 8) $\left.\left.\mathrm{C}_{6} \mathrm{H}_{5}, 9\right) 4-\mathrm{CH}_{3} \mathrm{O}-\mathrm{C}_{6} \mathrm{H}_{4}, 10\right) 4-\left(\mathrm{CH}_{3}\right)_{2} \mathrm{~N}-\mathrm{C}_{6} \mathrm{H}_{4}$;

$\mathbf{R}^{\prime}=$ 11) $\mathrm{CH}_{3}$, 12) $\mathrm{C}_{2} \mathrm{H}_{5}$.

Scheme 2 Synthesis of N-substituted benzimidazole derivatives 7-14

properties of the synthesized ethyl-[2-(1-(substituted phenyl)-5-oxopyrrolidinyl-3yl)- $1 H$-benzimidazolyl]etanoates have been investigated, and their benzimidazolium chlorides, 1-carboxymethylbenzimidazoles, have been obtained, and products of the condensation of 2-\{2-[1-(3-methylphenyl)-5-oxo-3-pyrrolidinyl]-1H-benzimidazol1 -yl $\}$ acetohydrazide with aromatic aldehydes and mono and diketones have been synthesized.

\section{Experimental}

The starting materials and solvents were obtained from Sigma-Aldrich Chemie (Germany) and Fluka (Switzerland) and were used without further purification. The methods used to follow the reactions were TLC and NMR. The NMR spectra were recorded on a Varian Unity Inova (300 MHz) spectrometer (Varian, USA). Chemical shifts are expressed as $\delta$, ppm relative to TMS. IR spectra $\left(v, \mathrm{~cm}^{-1}\right)$ were recorded on a Perkin Elmer BX FT-IR spectrometer (PerkinElmer, USA) using KBr tablets. Mass spectra were obtained on a Waters ZQ 2000 spectrometer (Waters, Germany) using the electrospray ionization (ESI) mode and operating at $25 \mathrm{~V}$. Elemental analyses were performed with a CE-440 elemental analyzer (Exeter Analytical, USA). Melting points were determined with a B-540 melting point analyzer (Büchi, USA) and are uncorrected. TLC was performed using Merck silica gel 60 F254 (Kieselgel 60 F254) plates. 
General procedure for preparation of benzimidazoles $\mathbf{2 a - 2 c}$

\section{Method A}

A mixture of the corresponding 1-substituted phenyl-4-carboxy-2-pyrrolidinone 1a-1c $(0.1 \mathrm{~mol})$ and 1,2 -diaminobenzene $(16.2 \mathrm{~g}, 0.15 \mathrm{~mol})$ was refluxed with hydrochloric acid $(4 \mathrm{M}, 80 \mathrm{ml})$ for $24 \mathrm{~h}$. The reaction mixture was cooled to the room temperature and neutralized with sodium hydroxide $(10 \%)$ up to $\mathrm{pH} 8-9$. The obtained solid was filtered off and washed with water. Products were purified by crystallizing from the corresponding solvent.

\section{Method B}

The corresponding amino acid $3(2 \mathrm{mmol})$ and $10 \mathrm{ml}$ of $10 \%$ hydrochloric acid were refluxed for $30 \mathrm{~min}$. Then, the reaction mixture was neutralized with aqueous ammonia to $\mathrm{pH}$ 8. The precipitated product was filtered off, washed with water, and dried.

\section{4-(1H-benzimidazol-2-yl)-1-(3-methylphenyl)pyrrolidin-2-one (2a)}

Yield 20.9 g (72 \%) (A), 0.55 g (94 \%) (B); m.p.: 182-183 ${ }^{\circ} \mathrm{C}$ (from 1,4-dioxane); ${ }^{1} \mathrm{H}$ NMR (300 MHz, DMSO- $\left.d_{6}\right): \delta=2.34\left(\mathrm{~s}, 3 \mathrm{H}, \mathrm{CH}_{3}\right), 2.97-3.15\left(\mathrm{~m}, 2 \mathrm{H}, 3-\mathrm{CH}_{2}\right)$, 4.01-4.19 (m, 1H, 4-CH), 4.25-4.38 (m, 2H, 5- $\left.\mathrm{CH}_{2}\right), 6.97-7.55$ (m, 8H, ArH), $11.61(\mathrm{~s}, 1 \mathrm{H}, \mathrm{NH}) \mathrm{ppm} ;{ }^{13} \mathrm{C} \mathrm{NMR}\left(75 \mathrm{MHz},\left(\mathrm{CD}_{3}\right)_{2} \mathrm{CO}\right): \delta=22.5\left(\mathrm{CH}_{3}\right), 33.0$ (4-C), 39.5 (3-C), 54.2 (5-C), 118.2, 118.5, 121.9, 121.9, 126.5, 126.5, 130.2, 140.0, 141.7 (ArC), $156.6(\mathrm{CN}), 173.5(\mathrm{CO}) ; \quad \mathrm{IR}(\mathrm{KBr}): \quad v=2,874(\mathrm{NH}), \quad 1,700$ (CO) $\mathrm{cm}^{-1}$; MS $(25 \mathrm{~V}): \mathrm{m} / z=292[\mathrm{M}+\mathrm{H}]^{+}(100)$; anal. calcd. for $\mathrm{C}_{18} \mathrm{H}_{17} \mathrm{~N}_{3} \mathrm{O}$, (\%): C, 74.21; H, 5.88; N, 14.42; found, (\%): C, 74.49; H, 5.81; N, 14.39.

\section{4-(1H-benzimidazol-2-yl)-1-(2,5-dimethylphenyl)pyrrolidin-2-one (2b)}

Yield 24.9 g (82 \%) (A), 0.56 g (91 \%) (B); m.p.: 260-261 ${ }^{\circ} \mathrm{C}$ (from dimethylformamide); ${ }^{1} \mathrm{H}$ NMR (300 MHz, DMSO- $\left.d_{6}\right): \delta=2.14$ (s, 3H, $\mathrm{CH}_{3}$ ), 2.27 (s, 3H, $\left.\mathrm{CH}_{3}\right), 3.04-3.18\left(\mathrm{~m}, 2 \mathrm{H}, 3-\mathrm{CH}_{2}\right), 4.13-4.28\left(\mathrm{~m}, 2 \mathrm{H}, 5-\mathrm{CH}_{2}\right), 4.44-4.52(\mathrm{~m}, 1 \mathrm{H}$, 4-CH), 7.06-7.82 (m, 7H, ArH) ppm; ${ }^{13} \mathrm{C}$ NMR (75 MHz, DMSO- $\left.d_{6}\right): \delta=17.1$ $\left(\mathrm{CH}_{3}\right), 20.2\left(\mathrm{CH}_{3}\right), 30.3$ (4-C), 35.3 (3-C), 52.8 (5-C), 113.8, 113.8, 125.4, 125.4, 127.1, 128.3, 130.4, 131.2, 132.2, 135.7, 136.7 (ArC), $153.5(\mathrm{CN}), 170.1$ (CO) ppm; IR $(\mathrm{KBr}): v=2,712(\mathrm{NH}), 1,687(\mathrm{CO}) \mathrm{cm}^{-1} ; \mathrm{MS}(25 \mathrm{~V}): \mathrm{m} / z=306[\mathrm{M}+\mathrm{H}]^{+}$ (100); anal. calcd. for $\mathrm{C}_{19} \mathrm{H}_{19} \mathrm{~N}_{3} \mathrm{O},(\%)$ : C, 74.73; H, 6.27; N, 13.76; found, (\%): $\mathrm{C}$, $74.59 ; \mathrm{H}, 6.51 ; \mathrm{N}, 13.59$.

\section{4-(1H-benzimidazol-2-yl)-1-(5-chloro-2-methylphenyl)pyrrolidin-2-one (2c)}

Yield 30.6 g (94 \%) (A), 0.62 g (95\%) (B); m.p.: 264-265 ${ }^{\circ} \mathrm{C}$ (from dimethylformamide); ${ }^{1} \mathrm{H}$ NMR (300 MHz, DMSO- $d_{6}$ ): $\delta=2.19$ (s, $3 \mathrm{H}, \mathrm{CH}_{3}$ ), 3.03-3.16 $\left(\mathrm{m}, 2 \mathrm{H}, 3-\mathrm{CH}_{2}\right), 4.16-4.32\left(\mathrm{~m}, 2 \mathrm{H}, 5-\mathrm{CH}_{2}\right), 4.49-4.62(\mathrm{~m}, 1 \mathrm{H}, 4-\mathrm{CH}), 7.33-7.80$ 
(m, 7H, ArH) ppm; ${ }^{13} \mathrm{C}$ NMR (75 MHz, DMSO-d $\left.d_{6}\right): \delta=17.1\left(\mathrm{CH}_{3}\right), 30.1$ (4-C), 35.3 (3-C), 52.7 (5-C), 113.8, 113.8, 125.4, 125.4, 126.7, 127.5, 130.1, 131.1, 132.1, 134.8, 138.3 (ArC), $153.5(\mathrm{CN}), 170.4(\mathrm{CO}) \mathrm{ppm}$; IR (KBr): $v=2,713(\mathrm{NH}), 1,704$ (CO) $\mathrm{cm}^{-1}$; MS $(25 \mathrm{~V}): \mathrm{m} / z=326[\mathrm{M}+\mathrm{H}]^{+}(100), 328[\mathrm{M}+2+\mathrm{H}]^{+}(50)$; anal. calcd. for $\mathrm{C}_{18} \mathrm{H}_{16} \mathrm{ClN}_{3} \mathrm{O}$, (\%): C, 66.36; H, 4.95; N, 12.90; found, (\%): C, 66.28; H, $4.21 ; \mathrm{N}, 12.62$.

General procedure for preparation of 3-(1H-benzimidazol-2-yl)-4-(substituted phenylamino)butanoic acids $\mathbf{3 a}-\mathbf{3 c}$

The corresponding substituted pyrrolidinone $\mathbf{2 a - 2 c}(5 \mathrm{mmol})$ was refluxed in sodium hydroxide solution $(20 \%, 20 \mathrm{ml})$ for $4 \mathrm{~h}$. After cooling, the reaction mixture was diluted with water to $50 \mathrm{ml}$, then filtered off, and the filtrate was acidified with acetic acid $(30 \%)$ to $\mathrm{pH} 6$. The precipitated product was filtered off, washed with water, and purified by dissolving the solid in a sodium hydroxide solution (5\%), filtering, and acidifying the filtrate with acetic acid (30\%).

\section{3-(1H-benzimidazol-2-yl)-4-(3-methylphenylamino)butanoic acid (3a)}

Yield $1.2 \mathrm{~g}$ (78 \%); m.p.: $187-188{ }^{\circ} \mathrm{C} ;{ }^{1} \mathrm{H}$ NMR (300 MHz, DMSO- $\left.d_{6}\right): \delta=2.16$ (s, 3H, $\left.\mathrm{CH}_{3}\right), 2.67-2.69\left(\mathrm{~m}, 2 \mathrm{H}, 2-\mathrm{CH}_{2}\right), 3.35-3.48\left(\mathrm{~m}, 2 \mathrm{H}, 4-\mathrm{CH}_{2}\right), 3.62-3.68(\mathrm{~m}$, $1 \mathrm{H}, 3-\mathrm{CH}$ ), 5.77 (br. s, 1H, NH), 6.33-7.50 (m, 8H, ArH), 12.74 (br. s, 1H, NH) ppm; ${ }^{13} \mathrm{C}$ NMR (75 MHz, DMSO- $\left.d_{6}\right): \delta=22.0\left(\mathrm{CH}_{3}\right), 36.9$ (3-C), $38.3(2-\mathrm{C}), 47.5$ (4-C), 110.0, 113.3, 115.2, 117.2, 121.6, 121.6, 129.4, 138.5, 139.4, 149.2 (ArC), $158.0(\mathrm{CN}), 175.3(\mathrm{CO})$ ppm; IR (KBr): $v=3,289(\mathrm{OH}), 2,852(\mathrm{NH}), 2,515(\mathrm{NH})$, 1,551 (CO) $\mathrm{cm}^{-1}$; anal. calcd. for $\mathrm{C}_{18} \mathrm{H}_{19} \mathrm{~N}_{3} \mathrm{O}_{2}$, (\%): C, 69.88; H, 6.19; N, 13.58; found, (\%): C, 69.79; H, 6.21; N, 13.69.

\section{3-(1H-benzimidazol-2-yl)-4-(2,5-dimethylphenylamino)butanoic acid (3b)}

Yield $1.2 \mathrm{~g}$ (75\%); m.p.: $240{ }^{\circ} \mathrm{C}$ (decomp.); ${ }^{1} \mathrm{H}$ NMR (300 MHz, DMSO- $\left.d_{6}\right)$ : $\delta=2.11\left(\mathrm{~s}, 3 \mathrm{H}, \mathrm{CH}_{3}\right), 2.28\left(\mathrm{~s}, 3 \mathrm{H}, \mathrm{CH}_{3}\right), 2.90-2.98\left(\mathrm{~m}, 2 \mathrm{H}, 2-\mathrm{CH}_{2}\right), 3.30-3.48(\mathrm{~m}$, $2 \mathrm{H}, 4-\mathrm{CH}_{2}$ ), 4.05-4.12 (m, 1H, 3-CH), 5.57 (br. s, 1H, NH), 7.14-7.53 (m, 7H, ArH), 13.25 (br. s., 1H, NH) ppm; IR (KBr): $v=3,003(\mathrm{OH}), 2,936(2 \mathrm{NH}), 1,579$ (CO) $\mathrm{cm}^{-1}$; anal. calcd. for $\mathrm{C}_{19} \mathrm{H}_{21} \mathrm{~N}_{3} \mathrm{O}_{2}$, (\%): C, 70.57; H, 6.55; N, 12.99; found, (\%): C, 70.49; H, 6.41; N, 13.19 .

\section{3-(1H-benzimidazol-2-yl)-4-(5-chloro-2-methylphenylamino)butanoic acid (3c)}

Yield 0.82 g (48 \%); m.p.: $222{ }^{\circ} \mathrm{C}$ (decomp.); ${ }^{1} \mathrm{H}$ NMR (300 MHz, DMSO- $\left.d_{6}\right)$ : $\delta=2.14\left(\mathrm{~s}, 3 \mathrm{H}, \mathrm{CH}_{3}\right), 2.70-2.81\left(\mathrm{~m}, 2 \mathrm{H}, 2-\mathrm{CH}_{2}\right), 3.32-3.48\left(\mathrm{~m}, 2 \mathrm{H}, 4-\mathrm{CH}_{2}\right)$, 3.60-3.65 (m, 1H, 3-CH), 5.98 (br. s, 1H, NH), 6.52-7.48 (m, 7H, ArH), 12.46 (br. s, $1 \mathrm{H}, \mathrm{NH}) \mathrm{ppm} ;{ }^{13} \mathrm{C} \mathrm{NMR}\left(75 \mathrm{MHz}, \mathrm{DMSO}-d_{6}\right): \delta=19.1\left(\mathrm{CH}_{3}\right), 36.5$ (3-C), 38.2 (2-C), 47.4 (4-C), 111.8, 112.4, 112.4, 115.2, 121.7, 121.8, 131.9, 134.3, 139.4, 139.5, 148.6 (ArC), $157.3(\mathrm{CN}), 174.7(\mathrm{CO}) \mathrm{ppm}$; IR (KBr): $v=3,434(\mathrm{OH}), 2,960$ 
(NH), 2,866 (NH), 1,603 (CO) $\mathrm{cm}^{-1}$; anal. calcd. for $\mathrm{C}_{18} \mathrm{H}_{18} \mathrm{ClN}_{3} \mathrm{O}_{2},(\%): \mathrm{C}, 62.88$; H, 5.28; N, 12.22; found, (\%): C, 62.38; H, 5.31; N, 12.42 .

General procedure for preparation of ethyl 2-\{2-[1-(substituted phenyl)-5-oxo-3pyrrolidinyl]-1H-benzimidazol-1-yl $\}$ acetates $\mathbf{4 a - 4 c}$

A mixture of the corresponding 1-aryl-3-(1H-benzimidazol-2-yl)-5-oxopyrrolidine 2a-2c $(0.01 \mathrm{~mol})$, potassium carbonate $(3.12 \mathrm{~g}, 20 \mathrm{mmol})$, potassium hydroxide powder $(1.12 \mathrm{~g}, 0.02 \mathrm{~mol})$, toluene $(40 \mathrm{ml})$, and tetrabutylammonium iodide $(0.1 \mathrm{~g})$ was heated to boiling, then, during 10 min stirring, chloroacetic acid ethyl ester $(6.6 \mathrm{ml}, 60 \mathrm{mmol})$ was added dropwise. The mixture was refluxed for $5 \mathrm{~h}$, then filtered hot. After cooling, the precipitated compound was filtered, washed with toluene, and crystallized from toluene.

Ethyl 2-\{2-[1-(3-methylphenyl)-5-oxo-3-pyrrolidinyl]-1H-benzimidazol-1yl $\}$ acetate (4a)

Yield 2.94 g (78 \%); m.p.: 146-147 ${ }^{\circ} \mathrm{C},{ }^{1} \mathrm{H}$ NMR (300 MHz, DMSO- $\left.d_{6}\right): \delta=1.23$ $\left(\mathrm{t}, J=7.1 \mathrm{~Hz}, 3 \mathrm{H}, \mathrm{CH}_{2} \mathrm{CH}_{3}\right), 2.32$ (s, 3H, $\left.\mathrm{CH}_{3}\right), 2.87-3.05$ (m, 2H, 4-C), 4.07-4.27 (m, 5H, 3-CH, 2- $\left.\mathrm{CH}_{2}, \mathrm{CH}_{2} \mathrm{CH}_{3}\right), 5.30\left(\mathrm{~s}, 2 \mathrm{H}, \mathrm{NCH}_{2}\right), 6.96-7.65$ (m, 8H, ArH) ppm; ${ }^{13} \mathrm{C}$ NMR (75 MHz, DMSO-d $): \delta=14.7\left(\mathrm{OCH}_{2} \mathrm{CH}_{3}\right), 21.9\left(\mathrm{CH}_{3}\right), 29.1$ (3-C), 38.3 (4-C), $45.1\left(\mathrm{NCH}_{2} \mathrm{CO}\right), 52.8$ (2-C), $62.1\left(\mathrm{OCH}_{2} \mathrm{CH}_{3}\right), 110.8,117.4,119.5$, 120.7, 122.6, 123.0, 125.5, 129.2, 136.4, 138.7, 139.9, 142.4 (ArC), 156.1 (CN), $169.1\left(\mathrm{NCH}_{2} \mathrm{CO}\right), 172.4(\mathrm{CO}) \mathrm{ppm}$; IR $(\mathrm{KBr}): v=1,735,1,692(\mathrm{CO}) \mathrm{cm}^{-1}$; MS $(25 \mathrm{~V}): m / z=378[\mathrm{M}+\mathrm{H}]^{+}(100)$; anal. calcd. for $\mathrm{C}_{22} \mathrm{H}_{23} \mathrm{~N}_{3} \mathrm{O}_{3},(\%): \mathrm{C}, 70.01 ; \mathrm{H}$, 6.14 ; N, 11.13; found, (\%): C, 70.19; H, 6.21; N, 11.29.

Ethyl 2-\{2-[1-(2,5-dimethylphenyl)-5-oxo-3-pyrrolidinyl]-1 $H$-benzimidazol-1yl $\}$ acetate $\mathbf{( 4 b )}$

Yield 2.35 g (60 \%); m.p.: 160-161 ${ }^{\circ} \mathrm{C} ;{ }^{1} \mathrm{H}$ NMR (300 MHz, DMSO-d $\left.d_{6}\right): \delta=1.21$ $\left(\mathrm{t}, J=7.1 \mathrm{~Hz}, 3 \mathrm{H}, \mathrm{CH}_{2} \mathrm{CH}_{3}\right), 2.13\left(\mathrm{~s}, 3 \mathrm{H}, \mathrm{CH}_{3}\right), 2.26\left(\mathrm{~s}, 3 \mathrm{H}, \mathrm{CH}_{3}\right), 2.87-2.91(\mathrm{~m}$, $2 \mathrm{H}, 4-\mathrm{C}), 3.97-4.25\left(\mathrm{~m}, 5 \mathrm{H}, 3-\mathrm{CH}, 2-\mathrm{CH}_{2}, \mathrm{CH}_{2} \mathrm{CH}_{3}\right), 5.30\left(\mathrm{~s}, 2 \mathrm{H}, \mathrm{NCH}_{2}\right)$, 7.06-7.87 (m, 8H, ArH) ppm; ${ }^{13} \mathrm{C}$ NMR (75 MHz, DMSO- $\left.d_{6}\right): \delta=14.7$ $\left(\mathrm{OCH}_{2} \mathrm{CH}_{3}\right), 17.9\left(\mathrm{CH}_{3}\right), 21.0\left(\mathrm{CH}_{3}\right), 30.3(3-\mathrm{C}), 36.9(4-\mathrm{C}), 45.0\left(\mathrm{NCH}_{2} \mathrm{CO}\right)$, 54.6 (2-C), $62.1\left(\mathrm{OCH}_{2} \mathrm{CH}_{3}\right), 110.8,119.5,122.6,123.0,127.7,128.8,131.22$, 131.5, 132.9, 136.4, 138.0, 142.5 (ArC), $156.4(\mathrm{CN}), 171.9\left(\mathrm{NCH}_{2} \mathrm{CO}\right), 172.7$ (CO) ppm; IR $(\mathrm{KBr}): v=1,731,1,690(\mathrm{CO}) \mathrm{cm}^{-1} ; \mathrm{MS}(25 \mathrm{~V}): \mathrm{m} / z=392[\mathrm{M}+\mathrm{H}]^{+}$ (100); anal. calcd. for $\mathrm{C}_{23} \mathrm{H}_{25} \mathrm{~N}_{3} \mathrm{O}_{3},(\%)$ : C, 70.57; H, 6.44; N, 10.73; found, (\%): $\mathrm{C}$, $70.29 ; \mathrm{H}, 6.41 ; \mathrm{N}, 10.59$.

Ethyl 2-\{2-[1-(5-chloro-2-methylphenyl)-5-oxo-3-pyrrolidinyl]-1Hbenzimidazol-1-yl $\}$ acetate $(\mathbf{4 c})$

Yield 2.14 g (52 \%); m.p.: 181-182 ${ }^{\circ} \mathrm{C} ;{ }^{1} \mathrm{H}$ NMR (300 MHz, DMSO- $\left.d_{6}\right): \delta=1.21$ $\left(\mathrm{t}, J=7.1 \mathrm{~Hz}, 3 \mathrm{H}, \mathrm{CH}_{2} \mathrm{CH}_{3}\right), 2.17\left(\mathrm{~s}, 3 \mathrm{H}, \mathrm{CH}_{3}\right), 2.90-2.93\left(\mathrm{~m}, 2 \mathrm{H}, 4-\mathrm{CH}_{2}\right)$, 
3.98-4.03 (m, 1H, 3-CH), 4.09-4.22 (m, 4H, 2- $\left.\mathrm{CH}_{2}, \mathrm{CH}_{2} \mathrm{CH}_{3}\right), 5.30$ (s, 2H, $\mathrm{NCH}_{2}$ ), 7.21-7.65 (m, 7H, ArH) ppm; ${ }^{13} \mathrm{C}$ NMR (75 MHz, DMSO- $\left.d_{6}\right): \delta=14.7$ $\left(\mathrm{OCH}_{2} \mathrm{CH}_{3}\right), 17.9\left(\mathrm{CH}_{3}\right), 30.5$ (3-C), 36.8 (4-C), $45.1\left(\mathrm{NCH}_{2} \mathrm{CO}\right), 54.3$ (2-C), $62.1\left(\mathrm{OCH}_{2} \mathrm{CH}_{3}\right), 110.8,119.5,122.2,123.5,127.2,128.0,130.9,132.9,135.4$, 136.4, 139.6, 142.5 (ArC), $156.2(\mathrm{CN}), 169.1\left(\mathrm{NCH}_{2} \mathrm{CO}\right), 172.2(\mathrm{CO}) \mathrm{ppm}$; IR $(\mathrm{KBr}): v=1,725,1,694(\mathrm{CO}) \mathrm{cm}^{-1}$; MS $(25 \mathrm{~V}): \mathrm{m} / z=412[\mathrm{M}+\mathrm{H}]^{+}(100), 414$ $[\mathrm{M}+2+\mathrm{H}]^{+}(50)$; anal. calcd. for $\mathrm{C}_{22} \mathrm{H}_{22} \mathrm{ClN}_{3} \mathrm{O}_{3},(\%)$ : C, 64.15; H, 5.38; N, 10.20; found, (\%): C, 64.38; H, 5.41; N, 10.32 .

General procedure for preparation of benzimidazolium chlorides $\mathbf{5 a - 5 c}$

A mixture of the corresponding ethyl ester $\mathbf{4 a}-\mathbf{4 c}(2.7 \mathrm{mmol})$ and concentrated hydrochloric acid $(10 \mathrm{ml})$ was refluxed for $4 \mathrm{~h}$. The reaction mixture was cooled, and the residue was filtered and washed with water.

1-(Carboxymethyl)-2-[1-(3-methylphenyl)-5-oxo-3-pyrrolidinyl]-1Hbenzimidazol-1-ium chloride (5a)

Yield $0.71 \mathrm{~g}(68 \%)$; m.p.: $158-159{ }^{\circ} \mathrm{C} ;{ }^{1} \mathrm{H}$ NMR (300 MHz, DMSO- $\left.d_{6}\right): \delta=2.32$ (s, 3H, $\left.\mathrm{CH}_{3}\right), 2.98-3.20\left(\mathrm{~m}, 2 \mathrm{H}, 4-\mathrm{CH}_{2}\right), 4.22-4.38\left(\mathrm{~m}, 2 \mathrm{H}, 2-\mathrm{CH}_{2}\right), 4.42-4.52$ (m, $1 \mathrm{H}, 3-\mathrm{CH}), 5.57$ (s, 2H, $\left.\mathrm{NCH}_{2} \mathrm{CO}\right), 7.22-7.98(\mathrm{~m}, 8 \mathrm{H}, \mathrm{ArH}) \mathrm{ppm} ;{ }^{13} \mathrm{C} \mathrm{NMR}$ (75 MHz, DMSO- $\left.d_{6}\right): \delta=21.9\left(\mathrm{CH}_{3}\right), 28.8$ (3-C), $38.0(4-\mathrm{C}), 46.4\left(\mathrm{NCH}_{2} \mathrm{CO}\right)$, 52.3 (3-C), 112.9, 116.0, 117.8, 121.1, 125.9, 126.0, 126.1, 129.3, 129.3, 133.6, 138.8, $139.5(\mathrm{ArC}), 155.2(\mathrm{CN}), 169.2\left(\mathrm{NCH}_{2} \mathrm{CO}\right), 171.2(\mathrm{CO}) \mathrm{ppm}$; IR (KBr): $v=3,333(\mathrm{OH}), 2,792\left(=\mathrm{N}^{+} \mathrm{H}-\right), 1,734,1,696(\mathrm{CO}) \mathrm{cm}^{-1}$; anal. calcd. for $\mathrm{C}_{20} \mathrm{H}_{20} \mathrm{ClN}_{3} \mathrm{O}_{3},(\%)$ : C, 62.26; H, 5.22; N, 10.89; found, (\%): C, 62.29; H, 5.31; N, 10.69 .

1-(Carboxymethyl)-2-[1-(2,5-dimethylphenyl)-5-oxo-3-pyrrolidinyl]-1Hbenzimidazol-1-ium chloride (5b)

Yield 0.86 g (80 \%); m.p.: $240{ }^{\circ} \mathrm{C}$ (decomp.); ${ }^{1} \mathrm{H}$ NMR (300 MHz, DMSO- $\left.d_{6}\right)$ : $\delta=2.15\left(\mathrm{~s}, 3 \mathrm{H}, \mathrm{CH}_{3}\right), 2.29\left(\mathrm{~s}, 3 \mathrm{H}, \mathrm{CH}_{3}\right), 2.91-2.94\left(\mathrm{~m}, 2 \mathrm{H}, 4-\mathrm{CH}_{2}\right), 3.98-4.10(\mathrm{~m}$, $2 \mathrm{H}, 2-\mathrm{CH}_{2}$ ), 4.18-4.27 (m, 1H, 3-CH), 5.24 (s, 2H, $\left.\mathrm{NCH}_{2} \mathrm{CO}\right), 7.08-7.29$ (m, 7H, ArH), 13.41 (br. s, $1 \mathrm{H}, \mathrm{COOH}) \mathrm{ppm} ;{ }^{13} \mathrm{C}$ NMR $\left(75 \mathrm{MHz}, \mathrm{DMSO}-d_{6}\right): \delta=17.9$ $\left(\mathrm{CH}_{3}\right), 21.0\left(\mathrm{CH}_{3}\right), 30.2(3-\mathrm{C}), 36.8(2-\mathrm{C}), 45.2\left(\mathrm{NCH}_{2} \mathrm{CO}\right), 54.5(2-\mathrm{C}), 111.0$, 119.1, 123.2, 123.3, 127.7, 128.89, 131.2, 132.9, 133.5, 136.5, 137.9 (ArC), 156.2 $(\mathrm{CN}), 170.4\left(\mathrm{NCH}_{2} \mathrm{CO}\right), 171.8(\mathrm{CO}) \mathrm{ppm}$; IR (KBr): $v=3,379(\mathrm{OH}), 2,922$ $\left(=\mathrm{N}^{+} \mathrm{H}-\right), 1,715,1,692(\mathrm{CO}) \mathrm{cm}^{-1}$; anal. calcd. for $\mathrm{C}_{21} \mathrm{H}_{22} \mathrm{ClN}_{3} \mathrm{O}_{3},(\%): \mathrm{C}, 63.08$; H, 5.55; N, 10.51; found, (\%): C, 63.29; H, 5.31; N, 10.69 .

1-(Carboxymethyl)-2-[1-(5-chloro-2-methylphenyl)-5-oxo-3-pyrrolidinyl]-1Hbenzimidazol-1-ium chloride $(\mathbf{5 c})$

Yield 0.58 g (51 \%); m.p.: $162-163{ }^{\circ} \mathrm{C} ;{ }^{1} \mathrm{H}$ NMR (300 MHz, DMSO- $\left.d_{6}\right): \delta=2.23$ (s, 3H, $\left.\mathrm{CH}_{3}\right), 2.91-3.21\left(\mathrm{~m}, 2 \mathrm{H}, 4-\mathrm{CH}_{2}\right), 4.08-4.27\left(\mathrm{~m}, 2 \mathrm{H}, 2-\mathrm{CH}_{2}\right), 4.56-4.62$ 
$(\mathrm{m}, 1 \mathrm{H}, 3-\mathrm{CH}), 5.51\left(\mathrm{~s}, 2 \mathrm{H}, \mathrm{NCH}_{2}\right), 7.36-7.92(\mathrm{~m}, 7 \mathrm{H}, \mathrm{ArH}) \mathrm{ppm} ;{ }^{13} \mathrm{C} \mathrm{NMR}$ (75 MHz, DMSO- $\left.d_{6}\right): \delta=17.9\left(\mathrm{CH}_{3}\right), 29.9$ (3-C), 36.7 (4-C), $46.3\left(\mathrm{NCH}_{2} \mathrm{CO}\right)$, 53.9 (2-C), 112.8, 116.1, 125.8, 126.0, 127.4, 128.3, 130.9, 132.9, 133.7, 135.6, 139.2 (ArC), $155.3(\mathrm{CN}), 169.3\left(\mathrm{NCH}_{2} \mathrm{CO}\right), 171.1(\mathrm{CO}) \mathrm{ppm}$; IR (KBr): $v=3,364$ $(\mathrm{OH}), 2,924\left(=\mathrm{N}^{+} \mathrm{H}-\right), 1,735,1,694(\mathrm{CO}) \mathrm{cm}^{-1}$; anal. calcd. for $\mathrm{C}_{20} \mathrm{H}_{19} \mathrm{Cl}_{2} \mathrm{~N}_{3} \mathrm{O}_{3}$, (\%): C, 57.16; H, 4.56; N, 10.00; found, (\%): C, 57.28; H, 4.51; N, 10.12 .

General procedure for preparation of acids $\mathbf{6 a - 6 c}$

Benzimidazolium chloride 5a-5c $(1 \mathrm{mmol})$ and sodium hydroxide solution $(5 \%$, $10 \mathrm{ml}$ ) were heated under reflux for $1 \mathrm{~min}$. The hot reaction mixture was acidified with acetic acid $(10 \%)$ to $\mathrm{pH} 6$ and left to cool. The residue was filtered off, washed with water, and purified by dissolving the solid in a sodium hydroxide solution (5\%), filtering the solution, and acidifying the filtrate with $10 \%$ acetic acid to $\mathrm{pH}$ 6.

2-\{2-[1-(3-Methylphenyl)-5-oxo-3-pyrrolidinyl]-1H-benzimidazol-1-yl $\}$ acetic acid (6a)

Yield $0.21 \mathrm{~g}(61 \%)$; m.p.: $278-279{ }^{\circ} \mathrm{C} ;{ }^{1} \mathrm{H}$ NMR (300 MHz, DMSO- $\left.d_{6}\right): \delta=2.32$ (s, 3H, $\mathrm{CH}_{3}$ ), 2.99-3.01 (m, 2H, 4- $\left.\mathrm{CH}_{2}\right), 4.00-4.05$ (m, 1H, 3-CH), 4.21-4.24 (m, $\left.2 \mathrm{H}, 2-\mathrm{CH}_{2}\right), 4.64\left(\mathrm{~s}, 2 \mathrm{H}, \mathrm{NCH}_{2}\right), 6.96-7.58$ (m, 8H, ArH) ppm; ${ }^{13} \mathrm{C}$ NMR $(75 \mathrm{MHz}$, DMSO- $\left.d_{6}\right): \delta=21.9\left(\mathrm{CH}_{3}\right), 29.3$ (3-C), 38.3 (4-C), 52.9 (2-C), 110.8, 117.4, 119.0, 120.7, 121.6, 122.0, 125.3, 129.2, 136.9, 138.6, 139.9, 142.4 (ArC), 156.4 (CN), $172.9(\mathrm{CO})$ ppm; IR (KBr): $v=3,374(\mathrm{OH}), 1,684,1,607(\mathrm{CO}) \mathrm{cm}^{-1}$; MS (25 V): $m / z=350[\mathrm{M}+\mathrm{H}]^{+}(100)$; anal. calcd. for $\mathrm{C}_{20} \mathrm{H}_{19} \mathrm{~N}_{3} \mathrm{O}_{3},(\%): \mathrm{C}, 68.75 ; \mathrm{H}, 5.48 ; \mathrm{N}$, 12.03; found, (\%): C, 68.59; H, 5.41; N, 12.09 .

2-\{2-[1-(2,5-Dimethylphenyl)-5-oxo-3-pyrrolidinyl]-1H-benzimidazol-1yl $\}$ acetic acid (6b)

Yield 0.22 g (61 \%); m.p.: 278-279 ${ }^{\circ} \mathrm{C} ;{ }^{1} \mathrm{H}$ NMR (300 MHz, DMSO- $\left.d_{6}\right): \delta=2.14$ (s, 3H, $\left.\mathrm{CH}_{3}\right), 2.29\left(\mathrm{~s}, 3 \mathrm{H}, \mathrm{CH}_{3}\right), 2.91-2.96\left(\mathrm{~m}, 2 \mathrm{H}, 4-\mathrm{CH}_{2}\right), 4.00-4.12(\mathrm{~m}, 3 \mathrm{H}$, 3-CH, 2- $\left.\mathrm{CH}_{2}\right), 4.57\left(\mathrm{~s}, 2 \mathrm{H}, \mathrm{NCH}_{2}\right), 7.09-7.61(\mathrm{~m}, 7 \mathrm{H}, \mathrm{ArH}) \mathrm{ppm} ;{ }^{13} \mathrm{C} \mathrm{NMR}$ (75 MHz, DMSO- $\left.d_{6}\right): \delta=17.9\left(\mathrm{CH}_{3}\right), 21.0\left(\mathrm{CH}_{3}\right), 30.6(3-\mathrm{C}), 36.8(4-\mathrm{C}), 54.7$ (2-C), 110.8, 119.0, 121.5, 122.0, 127.7, 128.7, 131.1, 132.9, 136.4, 137.0, 138.1, 142.5 (ArC), $156.5(\mathrm{CN}), 172.3(\mathrm{CO}) \mathrm{ppm}$; IR (KBr): $v=3,347(\mathrm{OH}), 1,679,1,605$ (CO) $\mathrm{cm}^{-1}$; MS (25 V): $\mathrm{m} / z=350[\mathrm{M}+\mathrm{H}]^{+}(100)$; anal. calcd. for $\mathrm{C}_{21} \mathrm{H}_{21} \mathrm{~N}_{3} \mathrm{O}_{3}$, (\%): C, 69.41; H, 5.82; N, 11.56; found, (\%): C, 69.69; H, 5.61; N, 11.49.

2-\{2-[1-(5-Choro-2-methylphenyl)-5-oxo-3-pyrrolidinyl]-1H-benzimidazol-1yl $\}$ acetic acid $(\mathbf{6 c})$

Yield 0.3 g (77 \%); m.p.: 216-217 ${ }^{\circ} \mathrm{C} ;{ }^{1} \mathrm{H}$ NMR (300 MHz, DMSO- $\left.d_{6}\right): \delta=2.16$ (s, 3H, $\mathrm{CH}_{3}$ ), 2.86-3.02 (m, 2H, 4- $\left.\mathrm{CH}_{2}\right), 4.03-4.13$ (m, 3H, 3-CH, 2- $\left.\mathrm{CH}_{2}\right), 4.78$ (s, $\left.2 \mathrm{H}, \mathrm{NCH}_{2}\right), 7.16-7.62(\mathrm{~m}, 7 \mathrm{H}, \mathrm{ArH}) \mathrm{ppm} ;{ }^{13} \mathrm{C} \mathrm{NMR}\left(75 \mathrm{MHz}, \mathrm{DMSO}-d_{6}\right)$ : 
$\delta=17.9\left(\mathrm{CH}_{3}\right), 30.7$ (3-C), 36.7 (4-C), 54.4 (2-C), 110.8, 119.1, 121.8, 122.3, 127.2, 127.9, 130.9, 132.8, 135.4, 136.8, 139.7, $142.5(\mathrm{ArC}), 156.3(\mathrm{CN}), 172.5$ (CO) ppm; IR (KBr): $v=3,386(\mathrm{OH}), 1,684,1,614(\mathrm{CO}) \mathrm{cm}^{-1}$; MS $(25 \mathrm{eV})$ : $m / z=384[\mathrm{M}+\mathrm{H}]^{+}(100), 386[\mathrm{M}+2+\mathrm{H}]^{+}$(50); anal. calcd. for $\mathrm{C}_{20} \mathrm{H}_{18} \mathrm{ClN}_{3} \mathrm{O}_{3}$, (\%): C, 62.58; H, 4.73; N, 10.95; found, (\%): C, 62.28; H, 4.51; N, 10.82.

\section{2-\{2-[1-(3-Methylphenyl)-5-oxo-3-pyrrolidinyl]-1 $H$-benzimidazol-1-} yl $\}$ acetohydrazide (7)

A mixture of ethyl ester $4 \mathbf{a}(3.77 \mathrm{~g}, 0.01 \mathrm{~mol})$, hydrazine hydrate $(3.4 \mathrm{~g}, 0.07 \mathrm{~mol})$, and 2-propanol $(60 \mathrm{ml})$ was refluxed for $2 \mathrm{~h}$. After cooling the reaction mixture to the ambient temperature, the precipitate was filtered off, washed with 2-propanol, and crystallized from dimethylformamide. Yield $2.28 \mathrm{~g}(63 \%) ;$ m.p.: $216-217{ }^{\circ} \mathrm{C}$; ${ }^{1} \mathrm{H}$ NMR (300 MHz, DMSO- $\left.d_{6}\right): \delta=2.34\left(3 \mathrm{H}, \mathrm{s}, \mathrm{CH}_{3}\right), 2.92-3.05\left(2 \mathrm{H}, \mathrm{m}, 4-\mathrm{CH}_{2}\right)$, 4.12-4.14 (1H, m, 3-CH), 4.22-4.28 (2H, m, 2- $\left.\mathrm{CH}_{2}\right), 4.63\left(2 \mathrm{H}, \mathrm{s}, \mathrm{NCH}_{2}\right), 4.94(2 \mathrm{H}$, $\left.\mathrm{s}, \mathrm{NH}_{2}\right), 6.96-7.52(8 \mathrm{H}, \mathrm{m}, \mathrm{ArH}), 9.64(1 \mathrm{H}, \mathrm{s}, \mathrm{NH}) \mathrm{ppm} ;{ }^{13} \mathrm{C} \mathrm{NMR}(75 \mathrm{MHz}$, DMSO- $\left.d_{6}\right): \delta=21.9\left(\mathrm{CH}_{3}\right), 29.2(3-\mathrm{C}), 30.9(4-\mathrm{C}), 45.1\left(\mathrm{NCH}_{2} \mathrm{CO}\right), 52.9$ (2-C), 110.7, 117.4, 119.4, 120.7, 122.4, 125.4, 129.2, 136.4, 138.6, 139.9, $142.4(\mathrm{ArC})$, $156.5(\mathrm{CN}), 166.7\left(\mathrm{NCH}_{2} \mathrm{CO}\right), 172.6(\mathrm{CO}) \mathrm{ppm}$; IR $(\mathrm{KBr}): v=3,299(\mathrm{NH}), 3,049$ $\left(\mathrm{NH}_{2}\right), 1,698,1,669(\mathrm{CO}) \mathrm{cm}^{-1}$; MS $(25 \mathrm{~V}): \mathrm{m} / z=364[\mathrm{M}+\mathrm{H}]^{+}$(100); anal. calcd. for $\mathrm{C}_{20} \mathrm{H}_{21} \mathrm{~N}_{5} \mathrm{O}_{2}$, (\%): C, 66.10; H, 5.82; N, 19.27; found, (\%): C, 66.59; H, 5.61; N, 19.09 .

General procedure for the synthesis of hydrazones 8-10

A mixture of hydrazide $7(0.73 \mathrm{~g}, 2 \mathrm{mmol})$, the corresponding aromatic aldehyde $(3 \mathrm{mmol})$, and ethanol $(30 \mathrm{ml})$ was refluxed for $5 \mathrm{~h}$. After cooling the reaction mixture to the ambient temperature, the precipitate was filtered off, washed with ethanol, and crystallized from dimethylformamide.

2-\{2-[1-(3-Methylphenyl)-5-oxo-3-pyrrolidinyl]-1 $H$-benzimidazol-1-yl $\}-N^{\prime}$ (phenylmethylidene)acetohydrazide (8)

Yield $0.69 \mathrm{~g}(76 \%)$; m.p.: $272-273{ }^{\circ} \mathrm{C} ;{ }^{1} \mathrm{H}$ NMR (300 MHz, DMSO- $\left.d_{6}\right): \delta=2.31$ $\left(3 \mathrm{H}, \mathrm{s}, \mathrm{CH}_{3}\right), 2.73-2.99\left(2 \mathrm{H}, \mathrm{m}, 4-\mathrm{CH}_{2}\right), 4.12-4.14(1 \mathrm{H}, \mathrm{m}, 3-\mathrm{CH}), 4.21-4.27(2 \mathrm{H}$, $\left.\mathrm{m}, 2-\mathrm{CH}_{2}\right), 5.15,5.62\left(2 \mathrm{H}, 2 \mathrm{~s}, \mathrm{NCH}_{2}\right), 6.96-7.83(13 \mathrm{H}, \mathrm{m}, \mathrm{ArH}), 8.11,8.30(1 \mathrm{H}$, $2 \mathrm{~s}, \mathrm{NCH}), 11.90,12.01(1 \mathrm{H}, 2 \mathrm{~s}, \mathrm{NH}) \mathrm{ppm} ;{ }^{13} \mathrm{C}$ NMR $\left(75 \mathrm{MHz}, \mathrm{DMSO}-d_{6}\right)$ : $\delta=21.9\left(\mathrm{CH}_{3}\right), 29.2(3-\mathrm{C}), 36.5(4-\mathrm{C}(\mathrm{Z})), 38.4(4-\mathrm{C}(E)) 44.9\left(\mathrm{NCH}_{2} \mathrm{CO}\right), 52.9$ (2-C), 110.8, 117.4, 119.4, 120.7, 122.3, 122.8, 125.4, 127.8, 127.9, 129.2, 129.5, 130.8, 134.6, 136.9, 138.6, 139.9, $142.5(\mathrm{ArC}), 145.1(\mathrm{NCH}), 156.7(\mathrm{CN}), 169.2$ $\left(\mathrm{NCH}_{2} \mathrm{CO}\right), 172.5(\mathrm{CO}) \mathrm{ppm}$; IR $(\mathrm{KBr}): v=3,099(\mathrm{NH}), 1,694,1,661(\mathrm{CO}) \mathrm{cm}^{-1}$; MS (25 V): $m / z=452[\mathrm{M}+\mathrm{H}]^{+}(100)$; anal. calcd. for $\mathrm{C}_{27} \mathrm{H}_{25} \mathrm{~N}_{5} \mathrm{O}_{2},(\%): \mathrm{C}, 71.82$; H, 5.58; N, 15.51; found, (\%): C, 71.59; H, 5.61; N, 15.49. 
$N^{\prime}$-[(4-methoxyphenyl)methylidene]-2-\{2-[1-(3-methylphenyl)-5-oxo-3pyrrolidinyl]-1H-benzimidazol-1-yl $\}$ acetohydrazide $(\mathbf{9})$

Yield $0.71 \mathrm{~g}(73 \%)$; m.p.: $236-237{ }^{\circ} \mathrm{C} ;{ }^{1} \mathrm{H}$ NMR (300 MHz, DMSO- $\left.d_{6}\right): \delta=2.31$ $\left(3 \mathrm{H}, \mathrm{s}, \mathrm{CH}_{3}\right), 2.93-3.04\left(2 \mathrm{H}, \mathrm{m}, 4-\mathrm{CH}_{2}\right), 3.81\left(3 \mathrm{H}, \mathrm{s}, \mathrm{OCH}_{3}\right), 4.09-4.14(1 \mathrm{H}, \mathrm{m}$, 3-CH), 4.19-4.27 (2H, m, 2- $\left.\mathrm{CH}_{2}\right), 5.12,5.59\left(2 \mathrm{H}, 2 \mathrm{~s}, \mathrm{NCH}_{2}\right), 6.93-7.95(12 \mathrm{H}, \mathrm{m}$, ArH), 8.05, $8.23(1 \mathrm{H}, 2 \mathrm{~s}, \mathrm{NCH}), 11.76,11.88(1 \mathrm{H}, 2 \mathrm{~s}, \mathrm{NH}) \mathrm{ppm} ;{ }^{13} \mathrm{C} \mathrm{NMR}$ (75 MHz, DMSO- $\left.d_{6}\right): \delta=21.9\left(\mathrm{CH}_{3}\right), 29.2(3-\mathrm{C},(Z)), 31.4(3-\mathrm{C},(E)) 36.5$ (4-C $(Z)), 38.4(4-\mathrm{C}(E)) 44.8\left(\mathrm{NCH}_{2} \mathrm{CO}\right), 52.9(2-\mathrm{C}), 56.0\left(\mathrm{OCH}_{3}\right), 110.8,115.0,115.0$, $117.4,117.4,120.7,122.3,122.8,125.4,127.2$, 129.2, 129.4, 129.5, 136.9, 138.6, 139.9, 142.5, $161.5(\mathrm{ArC}), 145.0(\mathrm{NCH}), 156.7(\mathrm{CN}), 163.0\left(\mathrm{NCH}_{2} \mathrm{CO}(Z)\right), 168.9$ $\left(\mathrm{NCH}_{2} \mathrm{CO}(E)\right), 172.5(\mathrm{CO})$ ppm; IR $(\mathrm{KBr}): v=3,124(\mathrm{NH}), 1,706,1,689(\mathrm{CO})$ $\mathrm{cm}^{-1}$; MS (25 V): $\mathrm{m} / z=482[\mathrm{M}+\mathrm{H}]^{+}(100)$; anal. calcd. for $\mathrm{C}_{28} \mathrm{H}_{27} \mathrm{~N}_{5} \mathrm{O}_{3},(\%): \mathrm{C}$, 69.84; H, 5.65; N, 14.54; found, (\%): C, 69.59; H, 5.61; N, 14.48.

$N^{\prime}$-\{[4-(dimethylamino)phenyl]methylidene $\}-2-\{2-[1-(3-m e t h y l p h e n y l)-5-o x o-$ 3-pyrrolidinyl]-1H-benzimidazol-1-yl acetohydrazide (10)

Yield 0.7 g (71 \%); m.p.: 210-211 ${ }^{\circ} \mathrm{C} ;{ }^{1} \mathrm{H}$ NMR (300 MHz, DMSO- $\left.d_{6}\right): \delta=2.31$ $\left(3 \mathrm{H}, \mathrm{s}, \mathrm{CH}_{3}\right), 2.95-3.01\left(8 \mathrm{H}, \mathrm{m}, 4-\mathrm{CH}_{2}+\mathrm{N}\left(\mathrm{CH}_{3}\right)_{2}\right), 4.11-4.15(1 \mathrm{H}, \mathrm{m}, 3-\mathrm{CH})$, 4.21-4.25 (2H, m, 2- $\left.\mathrm{CH}_{2}\right), 5.09,5.55\left(2 \mathrm{H}(0,29: 0,71), 2 \mathrm{~s}, \mathrm{NCH}_{2}\right), 6.74-7.62(12 \mathrm{H}$, $\mathrm{m}, \mathrm{ArH}), 7.98,8.14(1 \mathrm{H}, 2 \mathrm{~s}, \mathrm{NCH}), 11.61,11.72(1 \mathrm{H}, 2 \mathrm{~s}, \mathrm{NH}) \mathrm{ppm} ;{ }^{13} \mathrm{C} \mathrm{NMR}$ (75 MHz, DMSO- $\left.d_{6}\right): \delta=21.9\left(\mathrm{CH}_{3}\right), 29.2(3-\mathrm{C},(Z)), 31.4(3-\mathrm{C},(E)) 36.5$ (4-C $(Z)), 38.4(4-\mathrm{C}(E)), 39.7\left(\mathrm{~N}\left(\mathrm{CH}_{3}\right)_{2}\right), 44.8\left(\mathrm{NCH}_{2} \mathrm{CO}\right), 52.9$ (2-C), 110.8, 112.4, $112.4,117.4,119.4,120.7,121.9,122.3,122.8,125.4,129.1,129.2,136.9$, 138.6, 139.9, 139.95, 142.5, $151.4(\mathrm{ArC}), 146.0(\mathrm{NCH}), 156.7(\mathrm{CN}), 163.0\left(\mathrm{NCH}_{2} \mathrm{CO}(\mathrm{Z})\right)$, $168.5\left(\mathrm{NCH}_{2} \mathrm{CO}(E)\right), 172.6(\mathrm{CO}) \mathrm{ppm}$; IR $(\mathrm{KBr}): v=3,210(\mathrm{NH}), 1,689,1,672$ (CO) $\mathrm{cm}^{-1}$; MS $(25 \mathrm{~V}): \mathrm{m} / z=364[\mathrm{M}+\mathrm{H}]^{+}$(100); anal. calcd. for $\mathrm{C}_{29} \mathrm{H}_{30} \mathrm{~N}_{6} \mathrm{O}_{2}$, (\%): C, 70.43; H, 6.11; N, 16.99; found, (\%): C, 70.59; H, 6.21; N, 16.89.

General procedure for the synthesis of hydrazones 11, 12

A mixture of hydrazide $7(0.73 \mathrm{~g}, 2 \mathrm{mmol})$, acetone or methyl ethyl ketone $(30 \mathrm{ml})$ was refluxed for $7 \mathrm{~h}$. After cooling the reaction mixture to the ambient temperature, the precipitate was filtered off, washed with ethanol, and crystallized from the appropriate solvent.

$N^{\prime}$-(1-methylethylidene)-2-\{2-[1-(3-methylphenyl)-5-oxo-3-pyrrolidinyl]-1Hbenzimidazol-1-yl $\}$ acetohydrazide (11)

Yield $0.55 \mathrm{~g}(68 \%)$ m.p.: $210-211^{\circ} \mathrm{C}$ (from acetone); ${ }^{1} \mathrm{H}$ NMR (300 MHz, DMSO- $\left.d_{6}\right): \delta=1.93,1.95,1.96,2.01\left(6 \mathrm{H}, 4 \mathrm{~s},\left(\mathrm{CH}_{3}\right)_{2}\right), 2.31\left(3 \mathrm{H}, \mathrm{s}, \mathrm{CH}_{3}\right)$, 2.95-3.01 (2H, m, 4-CH $)$, 4.06-4.10 (1H, m, 3-CH), 4.18-4.25 (2H, m, 2- $\left.\mathrm{CH}_{2}\right)$, 5.14, 5.41 (2H (0.36:0.64), $\left.2 \mathrm{~s}, \mathrm{NCH}_{2}\right), 6.97-7.62(8 \mathrm{H}, \mathrm{m}, \mathrm{ArH}), 10.60,10.70(1 \mathrm{H}$ $(0,36: 0,64), 2 \mathrm{~s}, \mathrm{NH}) \mathrm{ppm} ;{ }^{13} \mathrm{C} \mathrm{NMR}\left(75 \mathrm{MHz}, \mathrm{DMSO}-d_{6}\right): \delta=17.2,17.7$ (cis (Z, E), $\left.\mathrm{C}\left(\mathrm{CH}_{3}\right)_{2}\right), 21.2\left(\mathrm{CH}_{3}\right), 24.9,25.2$ (trans $\left.(\mathrm{Z}, \mathrm{E}), \mathrm{C}\left(\mathrm{CH}_{3}\right)_{2}\right), 28.4$ (3-C), $37.6(4-\mathrm{C})$, 
44.4, $44.5\left(\mathrm{NCH}_{2} \mathrm{CO}\right), 52.2$ (2-C), 109.9, 116.6, 118.8, 120.0, 121.5, 122.0, 124.7,

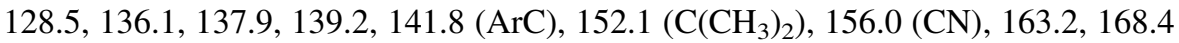
$\left(\mathrm{NCH}_{2} \mathrm{CO}\right), 171.8(\mathrm{CO}) \mathrm{ppm}$; IR $(\mathrm{KBr}): v=3,192(\mathrm{NH}), 1,699,1,677(\mathrm{CO}) \mathrm{cm}^{-1}$; MS (25 V): $m / z=404[\mathrm{M}+\mathrm{H}]^{+}(100)$; anal. calcd. for $\mathrm{C}_{23} \mathrm{H}_{25} \mathrm{~N}_{5} \mathrm{O}_{2},(\%): \mathrm{C}, 68.47$; H, 6.25; N, 17.36; found, (\%): C, 68.59; H, 6.21; N, 17.39 .

2-\{2-[1-(3-Methylphenyl)-5-oxo-3-pyrrolidinyl]-1H-benzimidazol-1-yl $\}-N^{\prime}-(1-$ methylpropylidene)acetohydrazide (12)

Yield $0.54 \mathrm{~g}$ (65\%); m.p.: $194-195{ }^{\circ} \mathrm{C}$ (from methyl ethyl ketone); ${ }^{1} \mathrm{H}$ NMR (300 MHz, DMSO- $\left.d_{6}\right): \delta=0.90,1.03,1.08,1.12$ (3H (E cis, Z trans, Z cis, E trans), $\left.4 \mathrm{t}, J=7.4 \mathrm{~Hz}, \mathrm{CCH}_{2} \mathrm{CH}_{3}\right), 1.91,1.94,1.95,2.07$ (3H (E cis, Z trans, $Z$ cis, E trans), $\left.4 \mathrm{~s}, \mathrm{CCH}_{3}\right), 2.22-2.44\left(5 \mathrm{H}, \mathrm{m}, \mathrm{CH}_{3}+\mathrm{CCH}_{2} \mathrm{CH}_{3}\right), 2.95-3.08\left(2 \mathrm{H}, \mathrm{m}, 4-\mathrm{CH}_{2}\right)$, $4.02-4.29\left(3 \mathrm{H}, \mathrm{m}, 3-\mathrm{CH}+2-\mathrm{CH}_{2}\right), 5.15,5.43\left(2 \mathrm{H}(0.34: 0.66), 2 \mathrm{~s}, \mathrm{NCH}_{2}\right)$, 6.96-7.64 (8H, m, ArH), 10.58, 10.64, 10.78, 10.80 (1H (0.24:0.08:0.52:0.16), $4 \mathrm{~s}$, (Z cis, Z trans, E cis, E trans) NH) ppm; IR (KBr), v, $\mathrm{cm}^{-1}: 3,207(\mathrm{NH}), 1,684,1,662$ (CO) $\mathrm{cm}^{-1}$; MS (25 V): $\mathrm{m} / z=418[\mathrm{M}+\mathrm{H}]^{+}$(100); anal. calcd. for $\mathrm{C}_{24} \mathrm{H}_{27} \mathrm{~N}_{5} \mathrm{O}_{2}$, (\%): C, 69.04; H, 6.52; N, 16.77; found, (\%): C, 69.29; H, 6.21; N, 16.79.

4-\{1-[2-(3,5-Dimethyl-1H-pyrazol-1-yl)-2-oxoethyl]-1H-benzimidazol-2-yl \}-1(3-methylphenyl)-2-pyrrolidinone (13)

A mixture of hydrazide 7 (0.73 g, $2 \mathrm{mmol}), 2$,4-pentanedione (0.8 g, $8 \mathrm{mmol})$, 2-propanol $(20 \mathrm{ml})$, and $\mathrm{HCl}(2$ drops) was refluxed for $5 \mathrm{~h}$. After cooling the reaction mixture to the ambient temperature, the precipitate was filtered off, washed with 2-propanol, and crystallized from 1,4-dioxane. Yield $0.46 \mathrm{~g}$ (53\%); m.p.: $138-139{ }^{\circ} \mathrm{C} ;{ }^{1} \mathrm{H}$ NMR $\left(300 \mathrm{MHz}, \mathrm{DMSO}-d_{6}\right): \delta=2.00\left(6 \mathrm{H}, \mathrm{s}, 3^{\prime}-\mathrm{CH}_{3}, 5^{\prime}-\mathrm{CH}_{3}\right)$, $2.33\left(3 \mathrm{H}, \mathrm{s}, \mathrm{CH}_{3}\right), 2.91-3.11\left(2 \mathrm{H}, \mathrm{m}, 3-\mathrm{CH}_{2}\right), 4.13-4.29\left(3 \mathrm{H}, \mathrm{m}, 4-\mathrm{CH}+5-\mathrm{CH}_{2}\right)$, 5.26, $5.32\left(2 \mathrm{H}, 2 \mathrm{~s}, \mathrm{NCH}_{2}\right), 6.01\left(\mathrm{H}, \mathrm{s}, 4^{\prime}-\mathrm{CH}\right), 6.99-7.69(8 \mathrm{H}, \mathrm{m}, \mathrm{ArH}) \mathrm{ppm}$; MS $(25 \mathrm{~V}): m / z=428[\mathrm{M}+\mathrm{H}]^{+}(100)$; anal. calcd. for $\mathrm{C}_{25} \mathrm{H}_{25} \mathrm{~N}_{5} \mathrm{O}_{2},(\%): \mathrm{C}, 70.24 ; \mathrm{H}$, 5.89; N, 16.38; found, (\%): C, 70.29; H, 5.71; N, 16.49 .

$N$-(2,5-dimethyl-1H-pyrrol-1-yl)-2-\{2-[1-(3-methylphenyl)-5-oxo-3pyrrolidinyl]-1 $H$-benzimidazol-1-yl acetamide (14)

A mixture of hydrazide 7 (0.73 g, $2 \mathrm{mmol})$, 2,5-hexanedione (1.14 g, $10 \mathrm{mmol})$, 2-propanol $(20 \mathrm{ml})$, and conc. acetic acid $(10 \mathrm{ml})$ was heated under reflux for $4 \mathrm{~h}$. The precipitate formed after cooling the reaction mixture to the ambient temperature was filtered off, washed with 2-propanol and crystallized from 2-propanol. Yield 0.59 g $(67 \%)$; m.p.: $204{ }^{\circ} \mathrm{C}$ (decomp.); ${ }^{1} \mathrm{H}$ NMR (300 MHz, DMSO- $\left.d_{6}\right): \delta=2.01$ $\left(6 \mathrm{H}, \mathrm{s}, 2^{\prime}-\mathrm{CH}_{3}, 5^{\prime}-\mathrm{CH}_{3}\right), 2.31\left(3 \mathrm{H}, \mathrm{s}, \mathrm{CH}_{3}\right), 2.91-3.11\left(2 \mathrm{H}, \mathrm{m}, 4-\mathrm{CH}_{2}\right), 4.14-4.32$ $\left(3 \mathrm{H}, \mathrm{m}, 3-\mathrm{CH}+2-\mathrm{CH}_{2}\right), 5.29\left(2 \mathrm{H}, \mathrm{s}, \mathrm{NCH}_{2}\right), 5.65\left(2 \mathrm{H}, \mathrm{s}, 3^{\prime}-\mathrm{CH}, 4^{\prime}-\mathrm{CH}\right), 6.96-7,66$ $(8 \mathrm{H}, \mathrm{m}, \mathrm{ArH}), 11.2(1 \mathrm{H}, \mathrm{s}, \mathrm{NH}) \mathrm{ppm} ;{ }^{13} \mathrm{C} \mathrm{NMR}\left(75 \mathrm{MHz}, \mathrm{DMSO}-d_{6}\right): \delta=11.0$ $\left(2^{\prime}-\mathrm{CH}_{3}, 5^{\prime}-\mathrm{CH}_{3}\right), 21.2\left(\mathrm{CH}_{3}\right), 28.6$ (3-C), 37.6 (4-C), $44.2\left(\mathrm{NCH}_{2} \mathrm{CO}\right), 52.1$ (2-C), 103.2 (3'-CH, 4'-CH), 109.7, 116.7, 118.9, 120.0, 121.9, 122.3, 124.7, 128.5, 135.7, 137.9, 139.1, 141.8 (ArC), 126.7 (2'-C, 5'-C), 155.68 (NC), $166.6\left(\mathrm{NCH}_{2} \mathrm{CO}\right), 171.7$ 
(CO) ppm; MS (25 V): $m / z=442[\mathrm{M}+\mathrm{H}]^{+}$(100); anal. calcd. for $\mathrm{C}_{26} \mathrm{H}_{27} \mathrm{~N}_{5} \mathrm{O}_{2}$, (\%): C, 70.73; H, 6.16; N, 15.86; found, (\%): C, 70.69; H, 6.11; N, 15.79.

Open Access This article is distributed under the terms of the Creative Commons Attribution License which permits any use, distribution, and reproduction in any medium, provided the original author(s) and the source are credited.

\section{References}

1. C. Kus, H. Goker, G. Ayhan, R. Ertan, N. Antanlar, A. Akin, Farmaco 51, 413 (1996)

2. H. Goker, E. Tebrizli, U. Abbasoglu, Arch. Pharm. 328, 53 (1995)

3. Y. Ozkay, Y. Tunali, H. Karaca, I. Isikdag, Eur. J. Med. Chem. 45, 3293 (2010)

4. B. Soni, M.S. Ranawat, A. Bhandari, P. Sharma, D. Choudhary, R. Sharma, R.P. Prajapat, Pharm. Glob. 3, 1 (2012)

5. C.J. Going, V.W. Mayer, Mutat. Res. 343, 185 (1995)

6. M.P. Nitin, P.T. Gowda, Int. Res. J. Pharm. 3, 189 (2012)

7. W.A. Maxwell, G. Brody, Appl. Microbiol. 21, 944 (1971)

8. M. Bretner, A. Baier, K. Kopanska, A. Najda, A. Schoof, M. Reinholz, A. Lipniacki, A. Piasek, T. Kulikowski, P. Borowski, Antivir. Chem. Chemother. 16, 315 (2005)

9. J. Valdez, R. Cedillo, A. Hernandez-Campos, L. Yepez, F. Hernandez-Luis, G. Navarrete-Vazquez, A. Tapia, R. Cortes, M. Hernandez, R. Castillo, Bioorg. Med. Chem. Lett. 12, 2221 (2002)

10. A.T. Mavrova, D. Vuchev, K. Anichina, N. Vassilev, Eur. J. Med. Chem. 45, 5856 (2010)

11. K. Kubo, Y. Inada, Y. Kohara, Y. Sugiura, M. Ojima, K. Itoh, Y. Furukawa, Y.K. Nishikawa, T. Naka, J. Med. Chem. 36, 1772 (1993)

12. F. Bordi, M. Mor, G. Morini, P.V. Plazzi, T. Silva, T. Vitali, A. Caretta, Farmaco 49, 153 (1994)

13. A. Satoh, T. Sagara, H. Sakoh, M. Hashimoto, H. Nakashima, T. Kato, Y. Goto, S. Mizutani, T. Azuma-Kanoh, T. Tani, S. Okuda, O. Okamoto, S. Ozaki, Y. Iwasawa, H. Ohta, H. Kawamoto, J. Med. Chem. 52, 4091 (2009)

14. D. Vásquez, C.F. Lagos, J. Mella-Raipán, L. González, R. Ebensperger, M.J. Alvarez-Figueroa, E. Sáez, H. Pessoa-Mahana, R. Araya-Secchp, A. González-Wong, T. Pérez-Acle, C.D. PessoaMahanavásquez, J. Chil. Chem. Soc. 52, 1281 (2007)

15. S.R. Zheng, S.L. Cai, J.B. Tan, J. Fan, W.G. Zhang, Inorg. Chem. Commun. 100, 1275 (2012)

16. J. Kulhanek, F. Bureš, Beilstein J. Org. Chem. 8, 25 (2012)

17. F. Saczewski, E. Dziemidowicz-Borys, P.J. Bednarski, R. Grunert, M. Gdaniec, P. Tabin, J. Inorg. Biochem. 100, 1389 (2006)

18. M. Mickevičius, Z. Beresnevičius, V. Mickevičius, G. Mikulskienè, Heteroat. Chem. 17, 47 (2006)

19. J.M. Miller, T.N. Ferraro, T.A. Hare, J. Neurochem. 55, 769 (1990)

20. K. Brokaité, V. Mickevičius, G. Mikulskiene, ARKIVOC ii, 61 (2006)

21. V. Mickevičius, R. Vaickelionienè, I. Jonuškienè, G. Mikulskienè, K. Kantminienè, Monatsh. Chem. 140, 1513 (2009) 